\title{
DIE GRIECHISCHEN \\ CHRISTLICHEN SCHRIFTSTELLER \\ DER ERSTEN JAHRHUNDERTE
}

\author{
PHILOSTORGIUS \\ KIRCHENGESCHICHTE
}





\title{
PHILOSTORGIUS \\ KIRCHENG ESCHICHTE
}

\section{Mit dem Leben des Lucian von Antiochien und den Fragmenten eines arianischen Historiographen}

\author{
HERAUSGEGEBEN \\ VON \\ JOSEPH BIDEZ
}

3., BEARBEITETE AUFLAGE

VON

FRIEDHELM WINKELMANN

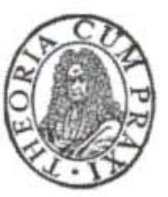

AKA DE M IE - VE RLAG - BERLIN 1981 


\section{Herauggegeben von}

Veselin Bě̌evliev, Ugo Bianchi, Alezander Böhlig, Hans Frhr. v. Campenhausen, Henry Chadwick, Ion Coman, Eligius Dekkers, Gerhand Delling,

Jacques Fontaine, Jḱnos Harmatta, Herbert Hunger, Johannes Irmocher (Verantwortlicher Herausgeber), Robert A. Kraft, Claude Mondésert, Marian Plezia, Harald Riesenfeld, Hans-Martin Schenke, Wilhelm Schneemelcher, Kurt Treu (Geschäftsführender Heransgeber), Ladislar Vidman

Mit Unterstützung des Zentralinstituts für Alte Geschichte und Archäologie der Akademie der Wissenschaften der DDR

Erachienen im Akademie-Verlag. DDR-1080 Berlin. Leipziger Str. 3-4 (C) Akademie-Verlag Berlin 1981

Lizenznummer : $202 \cdot 100 / 122 / 81$

Offetdruck und Bindung: VEB Druckerei „Thomas Müntzer", 5820 Bad I.angensalza Bestellnummer: $7536320(2031 / 18) \cdot$ LSV.6310

Printed in GDR

DDR 75,- $M$ 